\title{
Charge state dependence of cathodic vacuum arc ion energy and velocity distributions
}

Johanna Rosén

Applied and Plasma Physics, School of Physics (A28), University of Sydney, New South Wales 2006, Australia

Jochen M. Schneider

Materials Chemistry, RWTH Aachen University, D-52056 Aachen, Germany

André Anders

Lawrence Berkeley National Laboratory, Berkeley, California 94720, USA

\begin{abstract}
In the literature, conflicting conclusions are reported concerning the charge state dependence of cathodic arc ion energy and velocity distributions. It appears that data from electrostatic energy analyzers indicate charge state dependence of ion energy, whereas time-of-flight methods support charge state independence of ion velocity. Here we present charge-state-resolved ion energy distributions and calculate the corresponding ion velocity distributions in aluminum vacuum arc plasma. We show that the conflicting conclusions reported in the literature for the two different characterization techniques may originate from the commonly employed data interpretation of energy and velocity, in which peak values and average values are not carefully distinguished.
\end{abstract}


Cathodic vacuum arc plasma is well known to be highly ionized, with material dependent ion energy distributions (IEDs) extending up to several hundreds of $\mathrm{eV}$. $^{1}$ These properties are important for the synthesis of dense coatings, and may enable control of their structure evolution. ${ }^{2}$

Extensive studies concerning the ion energy or velocity have been performed, primarily dealing with the most likely ion velocity, ${ }^{3}$ the ion velocity distribution (IVD), ${ }^{1}$ or the IED ${ }^{4-7}$ generally averaged over all ion species present. Investigations that enable charge-state-resolved characterization usually involve time-of-flight (TOF) techniques ${ }^{8-11}$ and electrostatic field methods. ${ }^{12-19}$ Generally, reports based on TOF techniques indicate charge state independence of the velocity (distribution), ${ }^{8-11}$ whereas energy (distributions) obtained by electrostatic methods support the notion of charge state dependence. ${ }^{12-18}$

Literature reports where both the IEDs and the IVDs are presented for the same cathodic arc system are not available. In the current letter, a thorough analysis of measured ion energy as well as the calculated ion velocity is presented for a cathodic vacuum arc plasma using an aluminum cathode. We suggest that the conflicting conclusions in the literature may be understood based on choice of characterization method and corresponding data analysis. Furthermore, the results support a charge state dependence of the cathodic arc ion energy and velocity distribution functions.

For the present investigation, the charge-state-resolved IEDs for straight, nonfiltered plasma expansion were obtained with an electrostatic analyzer (PPM-422, Pfeiffer Vacuum), positioned $45 \mathrm{~cm}$ from the cathode. Data was acquired in vacuum at a base pressure of about $1 \cdot 10^{-4} \mathrm{~Pa}$. The arc was triggered by a flashover across a ceramic tube between a trigger electrode and the cathode, and powered by a direct current (dc) supply, with a resulting arc current of 35 A. To correct the measured 
IEDs for acceleration in the sheath at the grounded analyzer entrance, initial plasma characterization using a Langmuir probe was performed, showing a plasma potential of approximately $3 \mathrm{~V}$ with respect to ground. For further details of the experimental setup, see Ref. 15.

Fig. 1 shows the IEDs for $\mathrm{Al}^{1+}, \mathrm{Al}^{2+}$, and $\mathrm{Al}^{3+}$ (raw data in black, 100 point smoothening in white), corrected for sheath acceleration. The intensity of $\mathrm{Al}^{3+}$ is approximately $10 \%$ compared to those of $\mathrm{Al}^{1+}$ and $\mathrm{Al}^{2+}$, also evident from the level of noise. The presented IEDs are converted into IVDs, through $v=\sqrt{2 \cdot E / m}$, where $v, E$ and $m$ are the velocity, energy and ion mass, respectively. Additionally, the converted distribution intensity $I$ is corrected in every point to ensure conservation of the number of particles, with resulting IVDs as shown in Fig.1 (in grey). The intensity correction factor is calculated through the ratio between the integrated area between adjacent points in the IED, and the integrated area in the corresponding velocity interval in the IVD. Inserted in the figure are also the calculated (from a normalized distribution) average energy, $\langle E\rangle$,

$$
\langle E\rangle=\int I(E) \cdot E \cdot d E
$$

and the most probable energy, equal to the IED peak position, $E_{\text {peak }}$. Correspondingly, values of the average velocity, $\langle v\rangle$ and IVD peak position, $v_{\text {peak }}$, are also calculated and indicated. It can be seen that both $E_{\text {peak }}$ and $\langle E\rangle$ increase with increasing charge state, from 43 and $51 \mathrm{eV}$ for $\mathrm{Al}^{1+}$ to 55 and $119 \mathrm{eV}$ for $\mathrm{Al}^{3+}$, respectively. In the same way $v_{\text {peak }}$ and $\langle v\rangle$ change from 18000 and $18800 \mathrm{~m} / \mathrm{s}$ to 20000 and $27900 \mathrm{~m} / \mathrm{s}$.

The most likely velocity, $v_{\text {peak }}$, is in the literature often approximated to be equal to $\langle v\rangle$, which in turn is used to calculate $\langle E\rangle$. This, however, is correct only for 
Gaussian or other symmetric distributions. The shape of cathodic arc IEDs can generally be approximated by a shifted Maxwellian distribution ${ }^{12,15-16,20}$

$$
f(E)=C_{s}[E-\Delta E] \cdot \exp \left[-\left(\sqrt{E-\Delta E}-\sqrt{E_{d i r}}\right)^{2} / T\right]
$$

where $C_{s}$ is a scaling constant, $\Delta E$ is kinetic energy shift in the sheath, and $E_{d i r}$ is the directed center-of-mass ion energy (unit of energy and temperature $T$ here in $\mathrm{eV}$ ). A characteristic feature of the Maxwellian distribution is the high energy tail, which makes $\langle E\rangle>E_{\text {peak }}$, and similarly for the IVD, $\langle v\rangle>v_{\text {peak }}$. For a distinct distribution peak value (the most likely energy or velocity), the correlation between $v_{\text {peak }}$ and $E_{\text {peak }}$ is trivial. However, since $\langle E\rangle=m \cdot\left\langle v^{2}\right\rangle / 2$ (which is not equivalent to $\left.\langle E\rangle=m \cdot\langle v\rangle^{2} / 2\right)$, the average energy $\langle E\rangle$ can not be correlated to $\langle v\rangle$.

With the last section in mind, a brief summary of previously mentioned ion energy or velocity characterization methods permitting charge and mass resolution is presented: Ion velocity obtained with TOF techniques are generally based on peak location, where the drift time of a plasma flow is measured over a given distance. Hence, the most likely velocity is obtained (being less than the average velocity). Even when TOF is used to obtain IVDs, the focus is usually on the peak, due to scattered data. Electrostatic techniques can correspondingly give the most likely energy, though more common is scanning the energy for a fixed mass-to-charge ratio, to obtain the IEDs. These enable identification of relevant characteristics. In the ongoing debate concerning charge-state dependence of ion energy and velocity, investigations based on the electrostatic methods generally state higher energy for higher ion charge, as opposed to TOF studies that conclude independence. The conflicting conclusions based on the two different characterization techniques may be understood through the here presented analysis of both IEDs and IVDs. 
In Fig. 2, the energy and velocity differences in averages and distribution peak values between different charge states are shown. Since the focus is on the issue of possible charge-state dependence (and not on absolute values), all data are given normalized to the $\mathrm{Al}^{1+}$-value. The effect of a Maxwellian distribution shape (including the tail) is most pronounced for $\langle E\rangle$, where the values for $\mathrm{Al}^{2+}$ and $\mathrm{Al}^{3+}$ are 162 and $234 \%$ of the $\mathrm{Al}^{1+}$-value, respectively. Correspondingly, the $\langle v\rangle$-values are 125 and $148 \%$. Turning to the distribution peaks, the charge state dependence is not as pronounced: The values for the peak energy, 126 and $131 \%$ for $\mathrm{Al}^{2+}$ and $\mathrm{Al}^{3+}$, respectively, are still significantly different from $\mathrm{Al}^{1+}$. However, the peak velocity for $\mathrm{Al}^{2+}$ and $\mathrm{Al}^{3+}$ is within approximately $10 \%$ of the $\mathrm{Al}^{1+}$-value. Hence, considering the fluctuating character of the cathodic arc, no charge-state dependence can here be concluded based on $v_{\text {peak }}$ only. Concerning ion energy and velocity, we therefore argue that the charge-state dependence, generally observed in electrostatic measurements and at variance to conclusions based on TOF measurements, may be explained by the employed data interpretation.

The presented analysis supports a charge-state dependence of cathodic arc ion energy and velocity. However, in reports indicating similar conclusions, ${ }^{12-18}$ one still finds a spread in the energy data, and the dependence on charge is differently pronounced. Recent findings ${ }^{21}$ have shown that neutrals are present in cathodic arc plasmas, which may result in ion-neutral collisions with possible charge transfer. The neutrals may therefore contribute towards these observed differences. ${ }^{18}$ The analysis serve as to resolve some of the apparent conflicts in literature, and to suggest an answer to the question about a possible charge-state dependence of the ion energy/velocity. The results are of importance for an improved understanding of the 
ion acceleration mechanisms in cathodic vacuum arcs, and relevance for future progress in thin film growth modeling by energetic condensation.

In summary, we show that the conflicting conclusions reported in the literature concerning ion energy and velocity, as obtained with electrostatic versus TOF techniques, may originate from the commonly employed data interpretation of energy and velocity, in which peak values and average values are not carefully distinguished. The investigation supports a charge-state dependence of the ion energy and velocity, with higher energies for higher charge states.

This work was supported in part by the U.S. Department of Energy under Contract No. DE-AC02-05CH11231. J. R. acknowledges support from the Welch and Hans Werthén foundations, and J.M.S. gratefully acknowledges funding from DFG (Schn 735/9-1). 


\section{References:}

${ }^{1}$ E. Byon and A. Anders, J. Appl. Phys. 93, 1899 (2003)

${ }^{2}$ M. M. M. Bilek, M. Chhowalla, and W. I. Milne, Appl. Phys. Lett. 71, 1777 (1997)

${ }^{3}$ A. Anders and G. Yushkov, J. Appl. Phys. 91, 4824 (2002)

${ }^{4}$ M. M. M. Bilek, M. Chhowalla, M. Weiler, and W. I. Milne, J. Appl. Phys. 79, 1287 (1996)

${ }^{5}$ M. Chhowalla, C. A. Davis, M. Weiler, B. Kleinsorge, and G. A. J. Amaratunga, J. Appl. Phys. 79, 2237 (1996)

${ }^{6}$ C. Rusteberg, M. Lindmayer, B. Jüttner, and H. Pursch, IEEE Trans. Plasma Sci. 23, $909(1995)$

${ }^{7}$ A. A. Plyutto, V. N. Ryzhkov, and A. T. Kapin, Sov. Phys. JETP 20, 328 (1965)

${ }^{8}$ A. S. Bugaev, V. I. Gushenets, A. G. Nikolaev, E. M. Oks, and G. Y. Yushkov, Tech. Phys. 45, 1135 (2000)

${ }^{9}$ A. S. Bugaev, V. I. Gushenets, A. G. Nikolaev, E. M. Oks, and G. Y. Yushkov, IEEE Trans. Plasma Sci. 27, 882 (1999)

${ }^{10}$ K. Tsuruta, K. Sekiya, and G. Watanabe, IEEE Trans. Plasma Sci. 25, 603 (1997)

${ }^{11} \mathrm{G}$. Yushkov, Measurements of directed velocity in vacuum arc plasmas by arc current perturbation methods, Proc. XXth Int. Symp. Discharges and Electrical Insulation in Vacuum, Xi'an, P. R. China, 2000, p.260-263

${ }^{12}$ M. M. M. Bilek, P. J. Martin, D. R. McKenzie, J. Appl. Phys. 83, 2965 (1998)

${ }^{13}$ M. Chhowalla, Appl. Phys. Lett. 83, 1542 (2003)

${ }^{14}$ W. D. Davis and H. C. Miller, J. Appl. Phys. 40, 2212 (1969)

${ }^{15}$ J. Rosen, A. Anders, S. Mraz, and J. M. Schneider, J. Appl. Phys. 97, 103306 (2005)

${ }^{16}$ J. Kutzner and H. C. Miller, J. Phys. D: Appl. Phys. 25, 686 (1992)

${ }^{17}$ H. C. Miller, J. Appl. Phys. 52, 4523 (1981) 
${ }^{18}$ A. Anders and E. Oks, unpublished

${ }^{19}$ M. Galonska, R. Hollinger, and P. Spädtke, Rev. Sci. Instrum. 75, 1592 (2004)

${ }^{20}$ J. Rosen, A. Anders, S. Mráz, A. Atiser, and J. M. Schneider, J. Appl. Phys. 99, $123303(2006)$

${ }^{21}$ A. Anders, Appl. Phys. Lett. 85, 6137 (2004) 


\section{Figure captions:}

Figure 1: IEDs (in black) and IVDs (in gray) for $\mathrm{Al}^{1+}, \mathrm{Al}^{2+}$, and $\mathrm{Al}^{3+}$. Also inserted are the calculated (from the distribution) values of average energy and velocity, as well as the corresponding peak values of the energy and velocity distributions.

Figure 2: $\quad$ Summary of the charge state dependence of the calculated average energy and velocity $(\langle E\rangle$ and $\langle v\rangle)$, and distribution peak values $\left(E_{\text {peak }}\right.$ and $\left.v_{\text {peak }}\right)$, normalized to $\mathrm{Al}^{1+}$ values. The lines are drawn to guide the eye. 
Fig 1, Rosén et al, APL

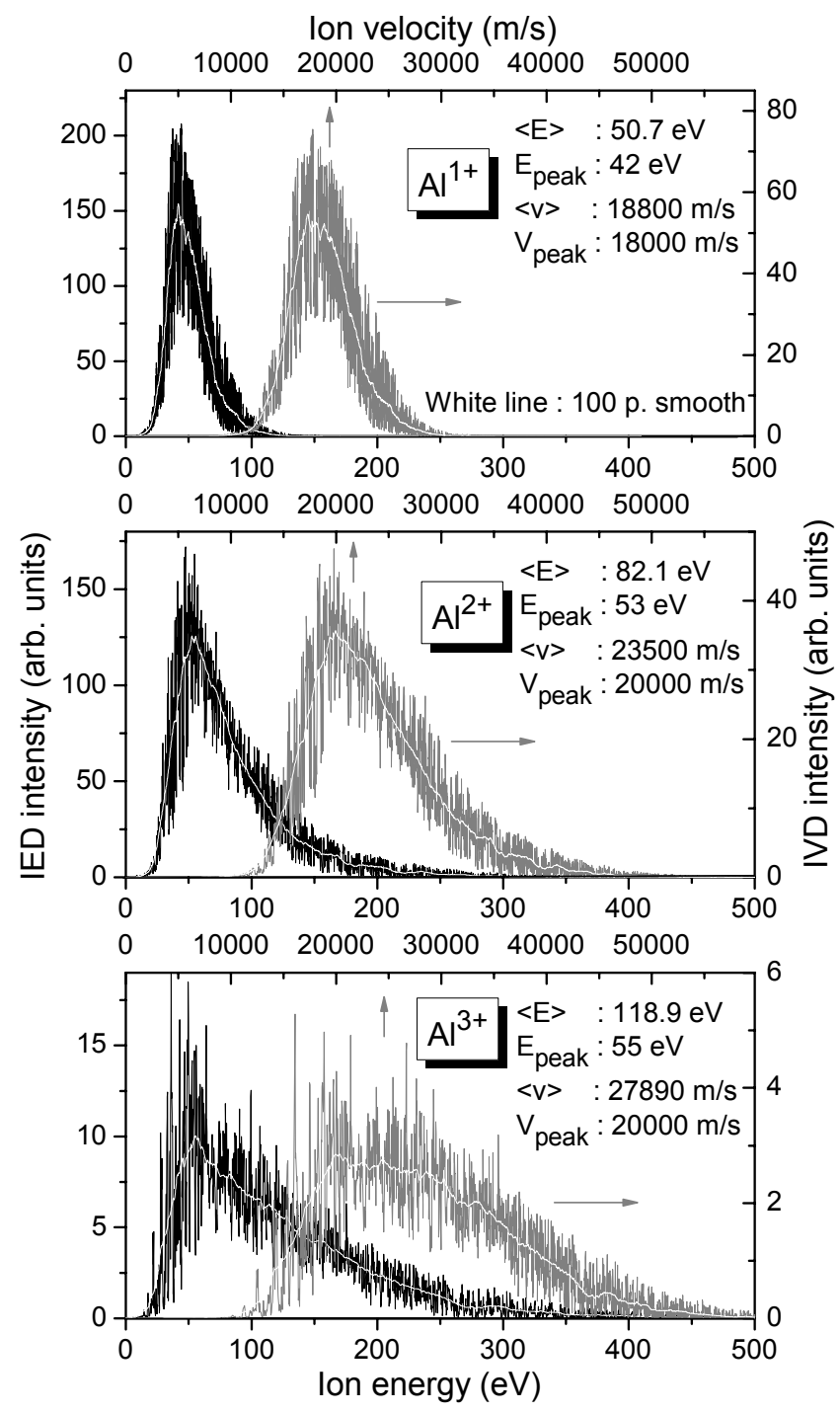


Fig 2, Rosén et al, APL

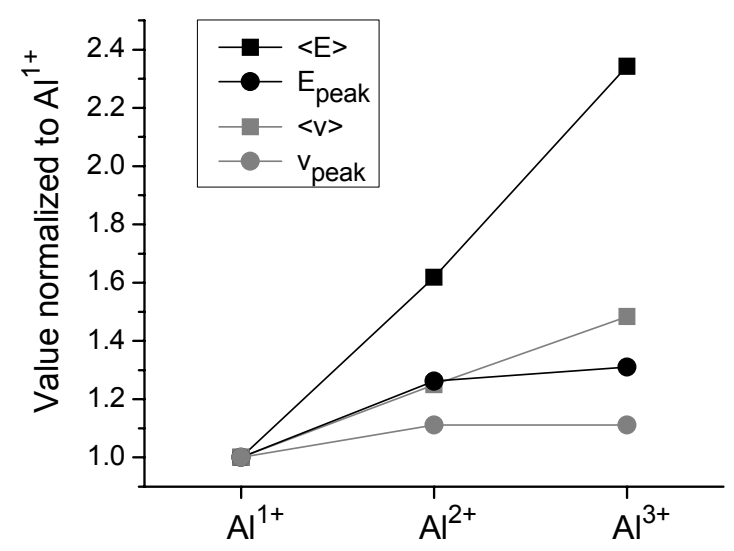

